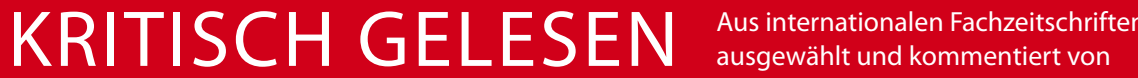

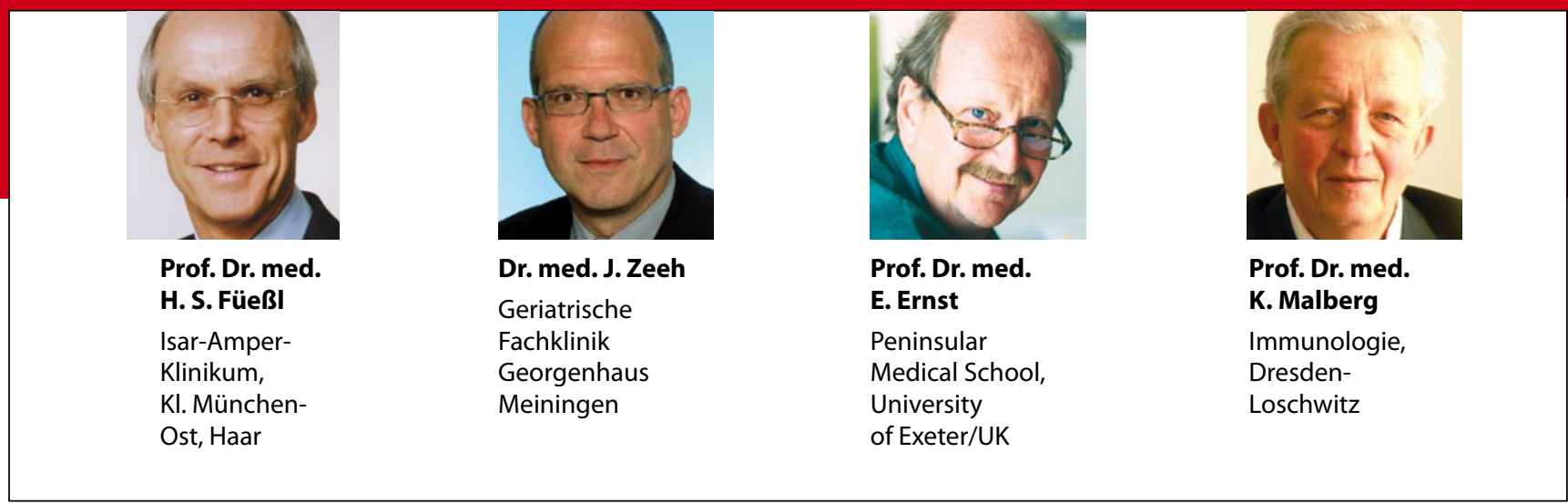

\section{Bisphosphonate verlängern die Überlebenszeit bei Osteoporose-Patientinnen}

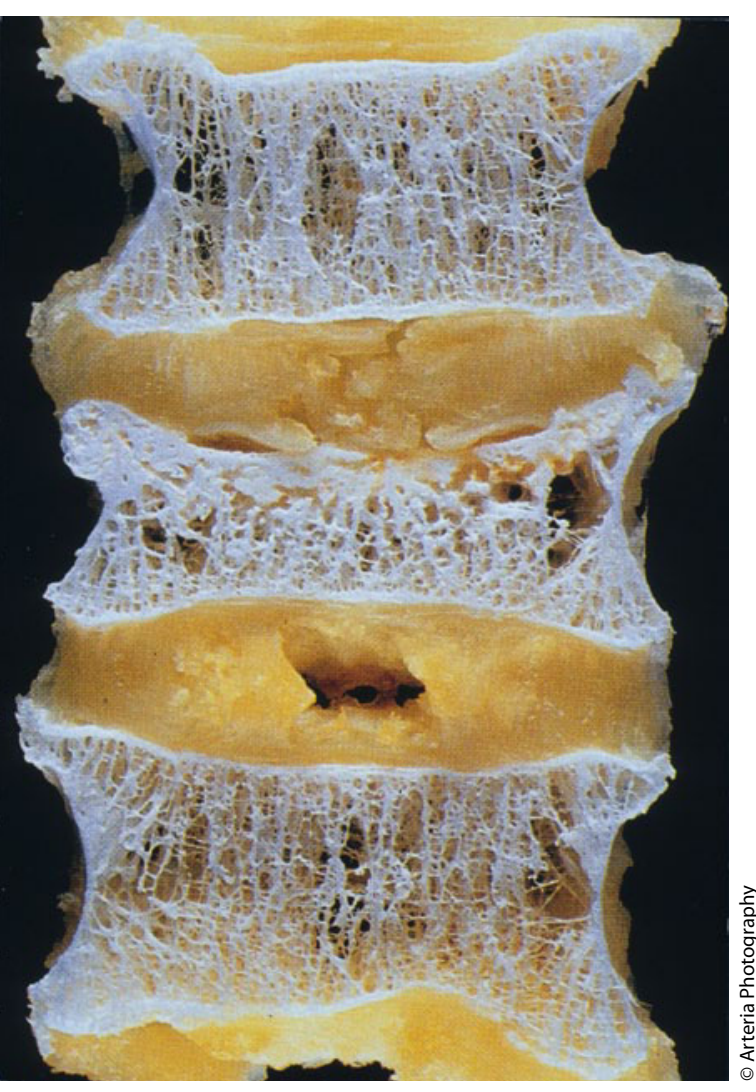

Osteoporose: Werden beim Knochenabbau Toxine freigesetzt?

\section{Osteoporotische Frakturen im} höheren Alter sind mit einer gesteigerten Mortalität verbunden. Eine antiresorptive Therapie reduziert die Frakturen und verlängert - zumindest bei Frauen - die Überlebenszeit signifikant.

- Forscher der University of New South Wales in Sydney (Australien) sammelten für eine prospektive Kohortenstudie die Daten von 2042 Probanden (1223 Frauen und 819 Männer, Alter 60 Jahre und älter, Studienbeginn 1989, Studienende 2007). Zweimal jährlich wurden bei diesen Personen die Knochendichte, der Gesundheitszustand und der Lebensstil erfasst. 325 Frauen und 37 Männer aus der Kohorte wurden wegen Osteoporose behandelt. 106 Frauen und 15 Männer nahmen Bisphosphonate ein, 142 Frauen und 22 Männer erhielten Kalzium plus Vitamin D und 77 Frauen bekamen Hormone.

Die Mortalitätsraten bei den Frauen betrugen 0,8/100 Patientenjahre in der Bisphosphonatgruppe, 1,2/100 Patientenjahre in der Hormongruppe, 3,2/100 Patientenjahre mit Kalzium plus Vitamin D und 3,5/100 Patientenjahre bei den Frauen, die keine Therapie er- hielten. Wurden konfundierende Faktoren herausgerechnet, blieb die Mortalität in der Bisphosphonatgruppe signifikant erniedrigt, nicht aber in der Hormongruppe. Auch bei den Männern unter Bisphosphonattherapie war die Mortalität verringert. Wegen der geringen Zahl an Probanden war der Effekt hier aber statistisch nicht signifikant.

\section{Kommentar}

Die Bisphosphonattherapie reduziert das Mortalitätsrisiko bei Frauen, wahrscheinlich auch bei Männern. Die Ursachen sind unklar. Die Autoren vermuten, dass Toxine, die im Laufe des Lebens in den Knochen gespeichert werden, beim altersbedingten erhöhtem Knochenabbau wieder freigegeben werden. Dieser Vorgang soll das Mortalitätsrisiko erhöhen. Bisphosphonate verhindern die Giftfreisetzung aus den Knochen und reduzieren so die Mortalität.

K. MALBERG

\section{- J. R. Center et al.}

(Garvan Institute of Medical Research, 384 Victoria Avenue, Darlinghurst, New South Wales 2010, Australia. E-mail: j.center@garvan.org.au) Osteoporosis medication and reduced mortality risk in elderly women and men. Published online on February 2, 2011, J. Clin. Endocrinol. \& Metab., doi:10.1210/jc.2010-2730 